\title{
Téoros
}

Revue de recherche en tourisme

\section{La ville de Québec : destination de congrès}

\section{Peter Gaudet et Pierre Lemay}

Volume 12, numéro 1, mars 1993

Québec, capitale et région touristiques

URI : https://id.erudit.org/iderudit/1078204ar

DOI : https://doi.org/10.7202/1078204ar

Aller au sommaire du numéro

Éditeur(s)

Université du Québec à Montréal

ISSN

0712-8657 (imprimé)

1923-2705 (numérique)

Découvrir la revue

Citer cet article

Gaudet, P. \& Lemay, P. (1993). La ville de Québec : destination de congrès.

Téoros, 12(1), 47-50. https://doi.org/10.7202/1078204ar d'utilisation que vous pouvez consulter en ligne.

https://apropos.erudit.org/fr/usagers/politique-dutilisation/ 


\title{
La ville de Québec Destination de congrès
}

\author{
Peter Gaudet et Pierre Lemay
}

\section{Introduction}

Capitale provinciale, la ville de Québec possède de nombreux atoutsquiluiontpermisd' acquérir une grande renommée touristique. Fondée en 1608 , Québec est la seule ville fortifíé de l'Amérique du Nord et le berceau de la civilisation française du continent. Elle offre un charme particulier du fait qu'elle a su conserver son cachet européen et son architecture unique tout en y intégrant des valeurs contemporaines pour en faire une ville où le passé et le présent se côtoient harmonieusement. Située sur les bords du majestueux fleuve SaintLaurent, la ville de Québec réussit à attirer annuellement des centaines de milliers de touristes qui désirent découvrir ses particularités culturelles. En 1985, l'UNESCO a déclaré l'arrondissement historique de Québec joyau du patrimoine mondial qui figure, depuis, à côté de Florence, de Rome, de Varsovie, de Jérusalem, etc. Seule ville en Amérique du Nord à être reconnue ainsi, cette nomination a contribué à l'établissement de la ville de Québec en tant que destination touristique importante.

Le tourisme decongrès occupe un créneau de plus en plus important dans l'industrie touristique de n'importe quelle ville. Nombreuses sont les études qui ont démontré l'ampleur des retombées économiques amenées par un congressiste comparativement à un touriste. On estime que le congressiste peut laisser derrière lui jusqu' au double de ce que dépense le touriste. Sans même tenir compte de la présence du conjoint, il n'est pas étonnant que tant d'importance soit accordée à l'élaboration d'une stratégie de mise en marché visant à attirer des congrès.

Au coeur de ce tourisme de congrès si convoité se trouvent les installations hôtelières et les salles de rassemblement. Offrant plus de 7500 chambres, Québec compteenviron 110 établissements hôteliers qui hébergent les clientèles d'affaires, d'agrément et de congrès. Parmi ceux-ci, 22 établissements, réunissant environ 4400 chambres et une super-

Massieurs Peter Gaudet et Pierre Lemay sont conseillers chez Caron, Bélanger, Ernst \& Young ficie totale de rassemblement de 173500 pieds carrés, servent, à des degrés divers, lemarché des congrès et des réunions. À ces installations hôtelières, s'ajoute actuellement une superficie de congrès de 45250 pieds carrés du Centre municipal de congrès de Québec.

C'est en 1974 que la ville de Québec s'est dotée du Centre municipal de congrès de Québec (CMCQ), établissant ainsi les fondations de sa renommée en tant que destination de congrès. L'inauguration d'un tel centre était relativement précoce au Canada, puisque seulement les villes de Saskatoon et de Calgary étaient ainsi pourvues. On peut donc considérer que la ville de Québec était alors une des pionnières de l'industrie des congrès au Canada, industrie qui n'a connu son plein essor qu'une décennie plus tard. Nous tenterons dans les paragraphes qui suivent de brosser un portrait général de cet important segment de l' industrie touristique de la ville de Québec.

\section{Le marché des congrès et des réunions}

La situation du marché des congrès et des réunions d'une ville dépend de plusieurs facteurs, Lanature des infrastructures locales, les caractéristiques socio-économiques, l'envergure des installations de congrès, la proximitédesmarchésciblesetl'accessibilité sont parmi les considérations premières dans la sélection d'un endroit pour la tenue d'un événement. Afin de bien cemer le marché des congrès, on doit différencier les divers événements qui amènent des retombées économiques importantes et qui utilisent parfois les infrastructures derassemblement, soit les congrès et les colloques, les expositions, les réunions de travail et les séances de formation, les foires commerciales, les salons pour consommateurs, et les événements locaux.

Les congrès et les colloques sont des rencontres privées de groupes ou d' associations professionnelles qui se tiennent habituellement dans les centres de congrès ou les hôtels munis d'installations propres à des activités de rassemblement. Le ministere du Tour- isme précise également à ce sujet que ces événements réunissent au moins 50 personnes ou plus dont la durée de séjour est supérieure à 24 heures (une nuit ou plus). Généralement, ces activités sont organisées une ou deux fois par année. Il arrive de plus en plus souvent que, dans le cadre d'un congrès ou d'une réunion, on tienne en parallèle une exposition grâce à laquelle on présente des produits et des services de l'organisation qui parraine l'événement.

Les réunions de travail sont des activités qui ont pour but la formation, l'information, la planification, l'évaluation et la délibération. Quant aux séances de formation, elles sont des périodes au cours desquelles les participants subissent des examens, suivent des cours ou font des apprentissages.

Les foires commerciales, a la différence des expositions dans le cadre de congrès et de colloques, tendent à privilégier l'aspect exposition en tant qu'événement et à tenir peu ou pas de rencontres ou de conférences en parallèle. On y présente des produits ou des services liés à un domaine d'activité précis et qui s"adressent généralement à tous les participants visés, et elles sont généralement présentées au même endroit année après année. Les salons pour consommateurs sont des événements qui s"adressent au grand public et comportent habituellement un droit d'admission. Ils servent à exposer des biens de consommation et se déroulent habituellement dans des centres de congrès ou des centres municipaux, selon leur importance et le type de marchandises qui y sont exposées. II existe enfin d'autres types d'événements qui se produisent dans des lieux de rassemblement publics et attirent un grand nombre de résidents. Ces événements comprennent lesconcerts, les activités à caractère social, culturel ou sportif, les banquets et autres types de rencontres.

Il y a autant de types d'événements de rassemblement que d'installations qui les accueillent, dont les hôtels, lescentres decongrès oucentresmunicipaux, les arénas, les centres de foires, etc. Aux fins de cet article, nous nous attarderons principalement aux événe- 
ments qui ont lieu dans les établissements hôteliers ou les centres de congrès.

Leshôtels ont longtemps été le lieu privilégié pour la tenue de congrès et de colloques. Par contre, l'envergure de leurs installations a eu tendance à freiner la demande potentielle du fait qu'ils posent certaines restrictions quant à la taille des événements possibles. Ainsi, bien que l'on puisse compter un total de plus de 110 hôtels dans la région immédiate de la ville de Québec, une vingtaine seulement offrent des salles de réunions. Ensemble, ces derniers réunissent un total de près de 200 salles où il est possible de tenir une réunion ou un congrès. D'après le Guide d'organisation de congrès, de réunions et de voyagesmotivation publie par l'Office du tourisme et des congrès de la Communauté urbaine de Québec et si l'on ne considêre que les hôtels qui disposent d'au moins une salle propre à accueillir 250 congressistes ou plus, cechiffre passe alors a dix, soit le Québec Hilton, l'Hồtel Loew's Le Concorde, Le Château Frontenac, le Holiday InnQuébec, le Radisson des Gouverneurs Québec, l'Hôtel des Gouvemeurs Sainte-Foy, l'Hôtel Plaza Universel, le Holiday Inn Sainte-Foy, l'Auberge Universel Wandlyn et l'Hôtel-Motel Universel. Quant aux centres de congrès, ils sont habituellement établis pour promouvoir et faciliter la tenue d'evénements de grande envergure et produire des retombées économiques au profit de la communauté en général. Les centres de congrès favorisent a priori la réservation de leurs installations aux événements de nature régionale, nationale et internationale qui ne s'adressent pas au public local. Ainsi, les retombées économiques sont maximisées par la venue des nombreux participants de l'extérieur. Un des objectifs d'un centre de congrès est d'agir en tant que complément aux installations disponibles au sein des infrastructures hôtelières en permettant la tenue de grands événements qui ne peuvent être reçus par un seul établissement hôtelier. Ainsi, les hôtels bénéficient de la demande accrue d'hébergement, et les commerces de la communauté hôte profitent des retombés ćconomiques crétes par l'arrivée de nombreux congressistes accompagnés de leurs conjoints. Ouvert en 1974 et troisième centre de congrès au Canada, le Centre municipal de congrès de Québec offre actuellement 15 salles de réunions dont la superficie totale est de 45250 pieds carrés. Laplus grandesalledisponible mesure 25645 pieds carrés et peut recevoir 3400 personnes pour une réunion ou 2640 personnes pour un banquet.
Pour leur part, les arénas, les centres de foires et autres endroits de rassemblement servent plus particulièrement à la tenue d'activités sportives, à des événements spéciaux ou à des manifestations culturelles. Ce genre d'installations a parfois le rôle secondaire d'être utilisé comme lieu d'exposition ou de foire, mais plutôt rarement comme lieu de congrès. Québec jouit de huit centres multidisciplinaires qui viennent compléter la brochette des endroits de rassemblement.

Comme nous l'avons mentionné précédemment, Québec compte, parmi ses infrastructures, le Centre municipal de congrès et, quoiqu'il ait été le sujet de nombreux débats au cours des demières années pour ce qui est de la gamme et de la qualité de ses installations, on doit tout de même reconnaître à ses promoteurs d'avoir devancé une tendance qui allait se manifester principalement au cours des années 1980. En fait, la ville de Québec a défini le besoin de se doter d'un centre de congrès en 1974, alors que les phénomènes de la multiplication de la demande de congrès et de la prolifération subséquente de centres de congrès plus spacieuxetplusmodemesn'allaientsemanifester qu'une décennie plus tard. De plus, le fait d'annexer leurs installations à un établissement hôtelier démontre la perspicacité des promoteurs de l'époque, puisque cette tendance s'est aussi manifestée seulement vers la deuxième moitié des années 80 et est reconnue aujourd'hui comme une formule plus avantageuse que l'aménagement de centres de congrès qui sont indépendants des établissements hôteliers.

\section{L'industrie des congrès à Québec}

Avant l' inauguration du Centre municipal de congrès de Québec en 1974, il n'existait, pour ainsi dire, aucune installation conçue exclusivement pour recevoir des réunions ou des congres, sauf les salles situces dans les hôtels, eten particulier, au Château Frontenac et au Loew's Le Concorde.

Avant 1970, la superficie totale disponible à Québec pour la tenue de réunions et de congrès d'un minimum de 250 personnes s'élevaità 48500 piedscarrés. Entre $1971 \mathrm{et}$ 1975 , cette superficie a plus que triplé avec l'ouverture du $\mathrm{CMCQ}$ et de cinq hôtels. Mais depuis, la région n'a vu qu'un seul nouveau lieu de congres, et cet ajout correspond à moins de $10 \%$ de la superficie totale actuellement disponible. II s'agissaitalors de l'ouverture de l'Hôtel Plaza Universel, qui amenait une superficie supplémentaire de 15700 pieds carrés sur le marché.

Compte tenu de la stagnation relative de l'offre, on constate que la capacité maximale pour un banquet est toujours de 2640 personnes depuis maintenant 15 ans. Comptant actuellement une superficie de 45250 pieds carrés, et selon une étude récente publice par la Ville de Québec ${ }^{(1)}$, le $C M C Q$ a vu se détériorer son classement pour ce qui est de la superficie de congrès et arrive aujourd'hui en dernière place parmi les principaux centres de congrès du Canada. Des dix centres analysés, cinq offraient le double de la superficie disponible au CMCQ.

Dans la région de Québec, le nombre de congrès et de congressistes est demeuré relativement stable depuis 1980. La moyenne annuelle entre 1980 et 1990 était d'environ 210 événements et 77300 participants. Au cours de la décennie, le plus faible nombre de participants était de 63789 en 1985, tandis que le nombre le plus élevétait de 98537 en 1981 (tableau 1).

En ce qui concerne l'origine des congrès tenus à Québec, le tableau 2 présente la répartition des congrès selon leur origine géographique entre 1985 et 1990 . On constate que presque lamoitié des 1250 congrès tenus à Québec provenaient de la province de Québec, environ $30 \%$, des autres provinces canadiennes, presque $10 \%$, des Etats-Unis, $3,5 \%$, de l'Amérique du Nord et $7,4 \%$, d'outre-mer.

La répartition des congrès tenus au Centre municipal de congrès de Québec en 1990 selon leur origine géographique contraste de façon frappante avec celle de la région de Québec. Les congrès d'origine provinciale représentaient $72 \%$ du total, ceux d'origine canadienne, $24 \%$, et les congrès internationaux, seulement $4 \%$. Onn'y comptaitaucun événement d'origine américaine en 1990. Cette répartition differe aussi de celle enregistrée au Palais des congrès de Montréal, où l'origine des congrès tenus en 1990-1991 se répartissait comme suit: $54,3 \%$ d'origine provinciale, $23,9 \%$ d'origine canadienne, $6,5 \%$ d'origine américaine et $15,2 \%$ d'origine internationale.

Il est intéressant de souligner que le $\mathrm{CMCQ}$ est celui qui bénéficie de la plus petite part de congrès d'origine américaine ou internationale. Ceci est particulièrement révélateur étant donné que le nombre de congrès internationaux tenus au Canada est passé de 93 à 

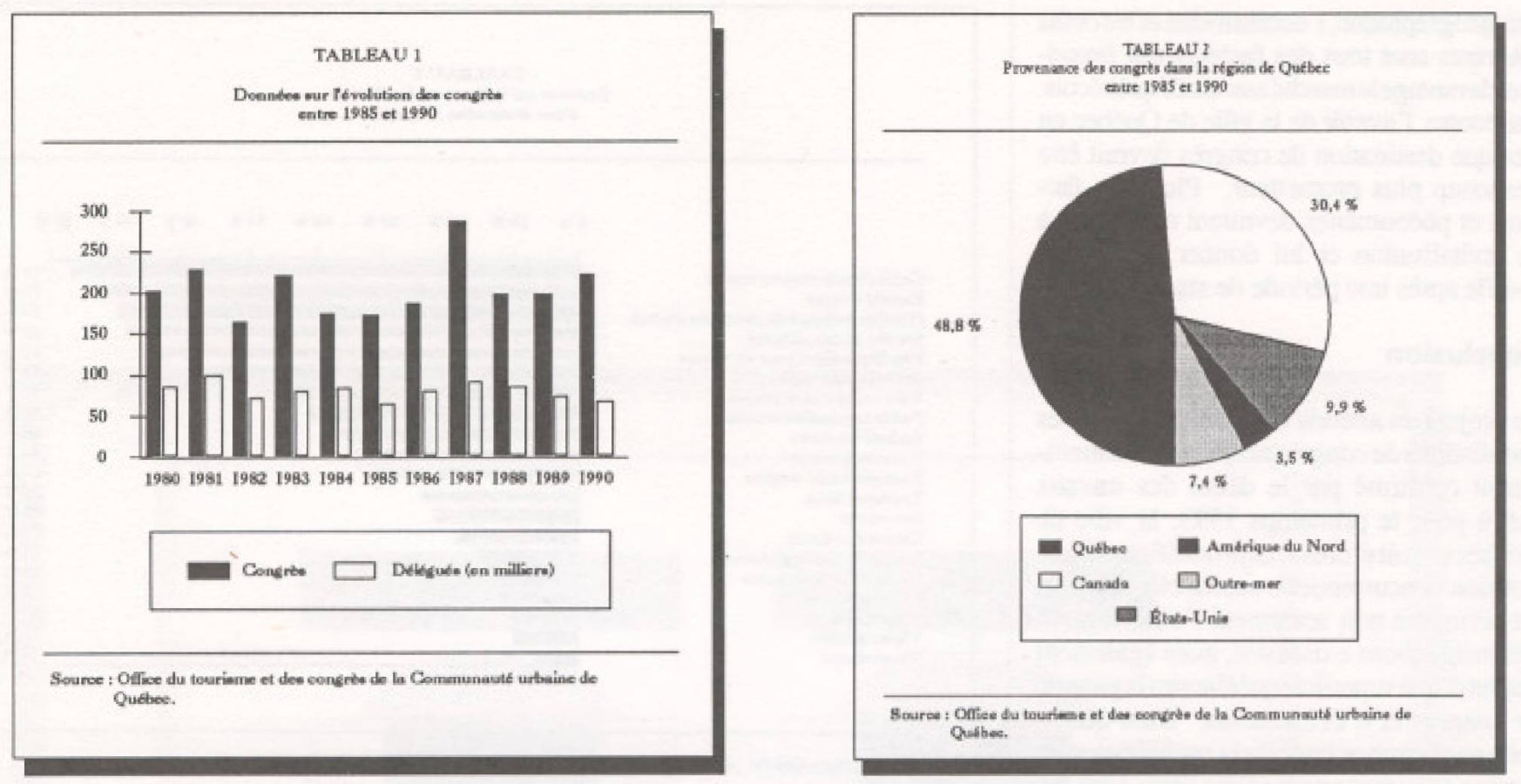

environ 300 entre 1975 et 1990 et que le nombre de congrès américains a augmenté en moyenne de $27 \%$ annuellement entre 1981 et $1987^{(2)}$. Comme les congrès de grandeenvergureproviennenthabituellement de ces marchés, on peut se demander si le CMCQ respecte ses objectifs d'attirer des événements qui auront des retombées pour l'ensemble des hôttels et des commerces de la municipalité plutôt que de bénéficier à un nombre restreint d'établissements. Cette faiblesse sur les marchés américain et international peut s'expliquer, entre autres, par la capacité relativement restreinte du centre, mais aussi par l'absence de liaisons aériennes directes entre Québecet les autres centres urbains nord-americains et étrangers.

Unautre facteurrévélateur au sein du marché des congrès de Québec est une certaine stagnation de la demande au CMCQ depuis 1980. Par contre, ce plafonnement a été atteint malgré le taux d'occupation relativement faible du centre, ce qui tend à souligner davantage l'insuffisance des installations actuelles. Pendant ce temps, le nombre de congrès dans la province de Québec a évolué au rythme annuel moyen de $12 \%$ entre 1984 et 1987 , et le nombre de congressistes a augmenté de $70 \%$ pendant cette période.

Nonseulement leCMCQ ne peut plus répondre à une demande grandissante, mais il doit aussi faire face a une concurrence accrue partout au pays. En effet, dix nouveaux centres ont ouvert au Canada depuis 1980 et ils ont le double de la superficie disponible au $\mathrm{CMCQ}^{(3)}$.

\section{Tendances du processus de sélection}

Afin de bien cerner la position actuelle de la ville de Québec en tant que destination de congrès, il importe de souligner les priorités et les tendances du processus de sélection d'un endroit pour la tenue d'un congrès. Pour ce faire, les études Metropoll réalisées conjointement par Economics Research Associates (ERA) et Survey Research Associates (SRA) auxÉtats-Unis sontd'excellents documents de référence. Celles-ci visent, à l'aide de sondages, à refléter la perception des principaux preneurs de décisions et organisateurs de réunions et de congrès en Amérique du Nord. Réalisées tous les trois ans, ces études évaluent plus de 50 villes nord-américainesen tant que lieux de congrès. Selon l'étude Metropoll IV, réalisée en $1991^{(4)}$, on constate d'abord que les organisateurs accordent beaucoup d'importance aux six facteurs suivants lors de l'évaluation d'un emplacement: les coûts liés àl'hébergement età la restauration, la facilitéd' accèsà la ville hôte pour les participants, la disponibilité d'hébergement, des services aériens adéquats, les frais de déplacement et l'attrait de l'hôtel (tableau 3).

Maintenant, si on compare l'image projetée par la ville de Québec en tant que destination de congrès dansl'étude Metropoll II de $1986^{(5)}$ en fonction des principaux critères des organisateurs de congrès aux Êtats-Unis, nous obtenons le classement suivant:

\begin{tabular}{lcc}
\hline & $\begin{array}{c}\text { Rang par rapport } \\
\text { a 55 lieux } \\
\text { nord-américains }\end{array}$ & $\begin{array}{c}\text { Rang par rapport } \\
\text { à 7lieus } \\
\text { canadiens }\end{array}$ \\
\hline $\begin{array}{l}\text { Coots lies a lhebergenent } \\
\text { et à la restauration }\end{array}$ & 28 & 1 \\
$\begin{array}{l}\text { Facilité daccès pour les } \\
\text { participants }\end{array}$ & 51 & 3 \\
$\begin{array}{l}\text { Disponbilité dhebergement } \\
\text { Services aériens adéquats }\end{array}$ & 35 & 1 \\
Frais de déplacement & 50 & 3 \\
\hline
\end{tabular}

Quoique le classement de la ville de Québec selon laperception des organisateurs démontre certaines faiblesses principalement au chapitre des frais de déplacement, de l'accessibilité et des services aériens, l'étude révèle que cette destination se voit mériter le $20^{\circ}$ rang quant à sa popularité. De plus, il semblerait que les efforts de marketing déployés atteignent le marché, puisque la notoriété publicitaire de Québec se classe $22^{*}$ parmi 65 destinations. Par ailleurs, la ville estreconnue pour la qualité de sa restauration et de ses installations de banquet. Malheureusement, ces attributs n'arrivent pas à compenser les lacunes de logistique, comme la facilité d'accès et les autres facteurs où Québec s'est vu attribuer de faibles classements quant à la capacité de ses installations. Compte tenu de ce qui précède, il n'est pas étonnant que la ville de Québec ait réussi a pénétrer davantage le marché des congrès québécois que les marchés américain et international. La capacité d"accueil, la situa- 
tion géographique, l'accessibilité et les coûtts inhérents sont tous des facteurs qui favorisentdavantage lemarché associatif québécois. Par contre, l'avenir de la ville de Québec en tant que destination de congrès devrait être beaucoup plus prometteur. Plusieurs facteurs et phénomènes devraient contribuer à sa revitalisation et lui donner un second souffle après une période de stagnation.

\section{Conclusion}

Le projet tant attendu d'agrandissement des installations de congrès actuellesétant maintenant confirmé par le début des travaux prévu pour le printemps 1993, la ville de Québecsevoitd'oresetdéjà bénéficierd'une position concurrentielle améliorée. Ce projet permettra non seulement l'amélioration des installations existantes, mais également l'ajoutd' une superficie quiélargira la gamme de congrès et d'événements. Bien que la forte concurrence issue de la prolifération de centres de congrès partout aux États-Unis, au Canada et même au Québec depuis le début des années 1980 ne soit pas éliminée, la ville de Québec pourra à nouveau se tailler une place enviable.

Puisque les retombées économiques du tourisme de congrès peuvent atteindre le double decelles amenées par le tourisme d'agrément, il n'est pas étonnant que tant de centres urbains investissentdans des installation pour satisfaire à la demande. Par contre, les installations à elles seules ne garantissent pas le succès dans ce domaine. La réalité confirme qu' une mise en marché dynamique et professionnelle, la connaissance des marchés cibles réels et le mariage de l'ensemble des services pour satisfaire les besoins des organisateurs sont les éléments qui contribueront davantage au succès.

\section{NOTES ET RÉFÉRENCES}

(1) Ville de Quebec, Pour un centre des congrest a la hauteur de notre potentiel, p. 50 .

(2) Idem, p. 10

(3) Idem, pp. 10 et 11.

(4) Economics Research Associates \& Survey Research Asscciates, Metropoll IV, Volume II. 1991, p. 57

(5) Economics Research Associates \& Survey Research Associates, Metropoill II, Québec City. 1986, Charts $\mathrm{A}$ and $\mathrm{B}$.
TABLEAU 3

Fecteurs qui influent aur la allection

frane deatianation de sondrts

Coúte (hốcela et reataurants)

Fasiliat d' uocots

Nombre muffiesnt de elenbra dhatel

Service arien adtequst

Pou Espendieux pouir ty rendre

Bel hotel de pongrèts

Ville ettrayante ef propre

Falble ariminalive wourit

Padroit populain

Bens restaurants

Bess centre de coasith

Tranaport kosel

Ben elimat

Cibyena andoaux

Bonate deatination familiale

Attrnotions touriatiquen

Uniquedtrirentant

Malasinas

Viaites fuidiles

Vie nosturne
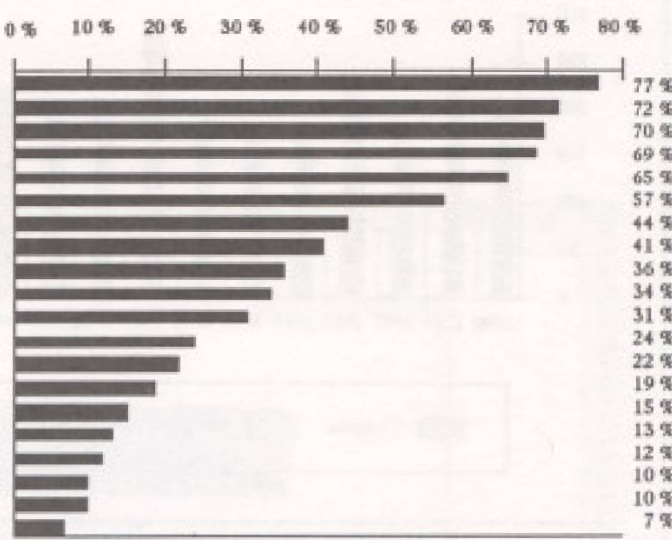

Source : Metropoll IV „Eeonomices Rewearch Ausceiaties, 1991

TABLEAU 4

Pereeption de la ville de Québee eomme destination de eongrès en comparaison avec 55 autres destinationa nord-américauines

Endroit populaire

Bonnes installations pour banquets

Qualitê de la nourriture

Coots moyena (hoteln et restaurants)

Peu dispendieux pour s'y nendre

Prix de loc ation

Serviabilite du personel

Nombre auffisant de chambres dhatel

Compétence de la direction

Proximité des hôtels de qualité

Service aérien adéquat

Facilité d'accés

Espaces suffisants pour expositions

Salles de rounions suffisantes

Nombre suffigant de places assisea

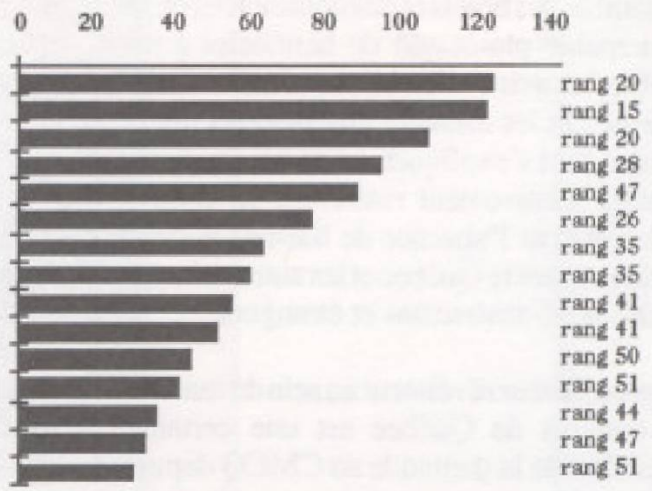

Source : Metropoll II , Economies Research Associntes, 1986 